\title{
TRASFORMAZIONI DELLO SPETTRO DELL' ARCO ELETTRICO CANTANTE
}

Memoria del Dott. M. LA ROSA.

In una nota preliminare ${ }^{2}$ ), pubblicata sui Rendiconti della R. Accademia dei Lincei, ho accennato alle modificazioni notevoli, che subisce lo spettro di un arco elettrico, quando viene posto nelle condizioni, in cui si ottiene il fenomeno dell' arco cantante, cioè quando ai poli dell'arco venga derivato un circuito di piccola resistenza, e dotato di capacità e d'induttanza. I valori delle costanti elettriche di questo circuito possono essere molto svariati; in particolare è possibile ridurre piccolissima la sua induttanza \%, in modo che esso non possieda più periodo proprio d'oscillazione.

Il fenomeno che si osserva in queste condizioni, differisce, per molti particolari, dall' arco cantante di Duddel ; si accosta invece a quei fenomeni ottenuti dal Prof. Righi ${ }^{3}$ ), derivando un condensatore ai poli di un tubo a vuoto, eccitato da una corrente continua ad alta tensione; e può essere riguardato come l'ultimo termine di una serie estesa di modificazioni graduali.

In questo lavoro sono riferiti più estesamente i risultati dello studio dello spettro emesso dall' arco cantante, in condizioni molto diverse, e sono poste in relazione le modificazioni spettrali con i principali elementi del fenomeno elettrico, al fine di trarne qualche utile conclusione sulle cause delle modificazioni medesime.

Le prime osservazioni furono fatte con uno spettroscopio Dubosq a due prismi, accuratamente tarato per mezzo dello spettro d'arco del mercurio, degli spettri dell' idrogeno e dell'elio,

1) Vol. XVII, ser, 5h, 1' sem., pag. 2no, 1908.

2) La Rnsa M., Kerd. Acr. Linc., XV1 pag. 112, 1907.

3) Righi, Reud. Acc. Bologna, Anu. 1901-02. 
e degli spettri di scintilla (con induttanza sufficiente per sopprimsro le righe doll' aria) fra elettrodi di cadmio, zinco, rame, piombo, stagno. Il riconoscimento delle righe di questi spettri fu fatto con l' aiuto dell' atlante spettrale dei sigg. Hagenbach e Konen. La curva di dispersione era sufficientemente esatta per la determinazione delle lunghezze d' onda fino ad una unità Angström; almeno nella regione più rifrangibile, in grazia della maggiore dispersione.

Accertate le modificazioni notevoli, che avvenivano nello spettro dell' arco, quando ai suoi poli si attaccava un conden. satore di grande capacità, intrapresi lo studio spettrografico, per poter acquistare una conoscenza sufficientemente esatta della natura e dell' entità di tali modificazioni.

Disposizione ottica. - La lampada ad arco da me impiegata aveva la forma di un ordinario spinterometro: cioè risultava di due colonnine di retro, che sostenevano i portacarboni; l'una fissa, l' altra mobile per mezzo di vite. Mercè un' opportuna disposizione si poteva, a piacere, fissare la posizione dell' arco lungo la verticale, su cui giacevano gli assi dei carboni.

Come spettrografo ho adoperato lo stesso apparecchio Dubosq, sostituendo pero al connocchiale una lente acromatica ( $\mathrm{L}$ in fig) di un metro di distanza focale. Al di lá di questa lente, era disposta una camera fotografica $18 \times 24$ (G), privata del suo obbiettivo. Essa era stata modificata in modo che il telaio posteriore, reso girevole attorno ad una normale alla base, poteva assumere, e mantenere invariata, qualunque inclinazione rispetto alla parete anteriore. Per mezzo di schermi adatti fu accuratamente esclusa dalla camera fotografica ogni traccia di luce falsa.

Un obbiettivo (0), di $14 \mathrm{~cm}$. di distanza focale, serviva a proiettare l'immagine dell' arco sulla fenditura del collimatore; esso era montato su un sostegno suscettibile di piccoli spostamenti in altezra e laterali.

Infine con una lente $\left(\mathrm{L}_{1}\right)$, di $5 \mathrm{~cm}$. di distanza focale, si pnteva fap convergere, fra i due carboni della lampada (A), un fascio di raggi solari, che penetrava nella stanza, attraverso 
ad un foro (B) di $5 \mathrm{~mm}$. di diametro, propagandosi nella direzione dell' asse del collimatore. Così, senza nulla mutare nella disposizione ottica impiegata per $\mathrm{l}^{+}$arco, si poteva facil-

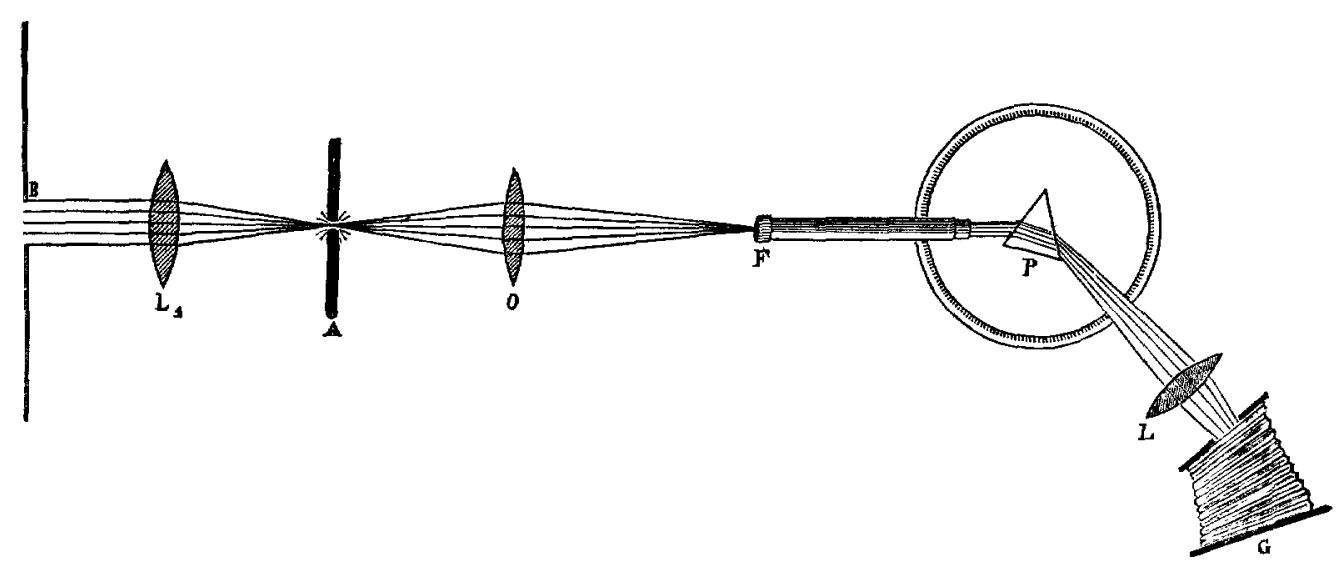

Fig. 1.

mente ottenere la fotografia dello spettro solarè, che serviva a tarare lo spettrografo.

Disposizione elettrica. - L' arco era prodotto fra due carboni omogenei, del diametro comune di $11 \mathrm{~mm}$,, disposti verticalmente; ed era alimentato da un circuito praticamente non induttivo, comprendente un reostato a lampade ed un amperometro, rilegato ai poli della rete elettrica urbana, con 1 ǒ0 volt di tensione.

Per ottenere il fenomeno oscillatorio si derivava ai poli di quest' arco, o un condensatore da 20 miclofarad, con le sottodivisioni 10 e 10 ; ovvero un altro condensatore da $10 \mathrm{mf}$., con le sottodivisioni $5,2,2,1$; ovvero l'uno e l'altro riuniti in superficie. Le connessioni erano fatte con due pezzi di cordoncino di rame (diametro $2 \mathrm{~mm}$ ), che insieme non raggiungevano la lunghezza di un metro; ed erano con ogni cura intrecciati, in modo da ridurre piccolissima la loro induttanza (non superiore ad un migliaio di centimetri). 
Per studiare lo spettro dell' arco nel regime di Duddel, si introduceva in serie col condensatore, o un rocchetto, con un solo strato di filo di rame, che aveva le costanti :

$$
\mathrm{R}=0,14 \mathrm{ohm} \quad \mathrm{L}=0,032 \cdot 10^{-3} \text { henry }
$$

ovvero un rocchetto a tre strati con le seguenti costanti;

$$
\begin{aligned}
& \text { strato } \quad 1^{\circ} \mathrm{R}=0,35 \mathrm{ohm} \quad \mathrm{L}=0,693.10^{-3} \text { henry } \\
& \text { 》 } 2^{0} 3^{0} \mathrm{R}=0,66 \gg \mathrm{L}=2,317 \text { 》 } \gg \\
& \text { » } 1^{\circ} 2^{6} 3^{0} \mathrm{R}=1,01 \text { » } \mathrm{L}=5,227 \text { 》 》 }
\end{aligned}
$$

Infine, per confrontare gli spettri dell' arco ottenuti nelle diverse condizioni del circuito derivato ai suoi poli, con lo spettro della scintilla fra $i$ medesimi pezzi di carbone, si toglievano le comunicazioni del circuito elettrico sopra detto con la lampada ad arco, e si pilegavano i due carboni alle armature di due bottiglie di Leyda, ciascuna di $500 \mathrm{~cm}$. circa di capacità, riunite in superficie.

Questo condensatore era caricato con un rocchetto d' induzione da $15 \mathrm{~cm}$. di scintilla, eccitato da una corrente di 6 amp., interrotta da un interruttore Foucault, circa 10 volte per secondo. Nel circuito di carica era introdotta una scintilla di $3 \mathrm{~cm}$.; la distanza esplosiva fra i due carboni era $0,3 \mathrm{~cm}$. circa.

Studio ottico. - Col procedimento comunemente impiegato (del cannocchiale adattato a distanza infinita), fu portata la fenditura esattamente nel piano focale dell' obbiettivo del collimatore; e con l' aiuto di un piccolo schermo di carta (su cui erano segnati due diametri ortogonali, uno dei quali parallelo ai bordi della fenditura), ben adattato su questo, si dispose l' asse del collimatore esattamente nella direzione del fascio solare che penetrava dal foro $B$.

Sul cammino del fascio furono quindi interposti la lente $L_{1}$ e l'obbiettivo $O$, nella posizione conveniente, perchè l'immagine del sole si formasse ben centrata sul piano della fenditura. Le distanze tra la fenditura e l'obbiettivo $O$, e tra questo e l'arco, erano state stabilite in modo, che l'imagine di questo fosse all' incirca tre volte il vero; ció per ottenere che attraverso alla fenditura, di cui l'altezza era stata ri- 
dotta a $2 \mathrm{~mm}$. circa, penetrasse esclusivamente la luce proveniente dall' arco (e non quella dei carboni), anche quando questo arera appena la lunghezza di un millimetro.

Fissate le posizioni di $L_{1}$ e di $O$, era facile tenere l'arco esattamente sull' asse comune di queste lenti e del collimatore.

Finalmente si disponeva, nella direzione dell' asse del fascio emergente dai prisini, l'asse ottico della lente $\mathrm{L}$, che serviva a proiettare lo spettro sulla lastra di vetro della camera fotografica.

Questa lastra era trasparente, e le imagini formate sulla sua faccia anteriore, venivano osservate mediante un oculare, adattato per la netta visione di una sottile linea incisa su questa faccia.

La posizione di questa lastra si stabiliva osservando lo spettro solare; poichè le linee di Fraunhofer', a motivo della loro grande sottigliezza, si prestavano assai meglio delle righe e bande luminose dello spettro dell arco.

Nella maggior parte delle fotografie (ed in tutte quelle riprodotte) furono usate lastre ortoscopiche Jougla, in mancanza delle Perutz specialmente consigliate; esse furono trattate con rivelator'e all' jdrammina diluito, che in virtù della sua lentezza permetteva la correzione dei difetti di posa, dentro limiti abbastanza ampi.

La regione spettrale fotografata è compresa fra le lunghezze d'onda $\lambda=5896$, e $\lambda=3964$ (D ed $\mathrm{H}$ dello spettro solare). Peró, a causa della notevole dispersione, le prove furono fatte in due tempi: in un primo fu fotografata la regione fra le lunghezze d'onda 5896 e 4530, ed in un secondo quella fra 473') e 3964. Per ciascuna regione fu presa una numerosa serie di fotografie, corrispondenti a condizioni molto svariate dell' arco, ed alcune alla scintilla fra i medesimi pezzi di carbone.

Ciascuna serie di fotografie fu preceduta e seguita dalla riproluzione della corrispondente regione dello spettro solare, almeno in doppio esemplare: ciò serviva, oltre che per i necessari confronti, per assicurarsi che inalterate erano rimaste le posizioni delle diverse parti del sistema spettrografico, nella riproduzione degli spettri di una stessa serie. 
Nella tavola I sono riprodotte alcune fotografie della prima regione, e nella tavola II le corrispondenti della seconda, disposte nel medesino ordine. Quelle coi numeri 27,58 rappresentano lo spettro dell' arco continuo, quelle coi numeri 43 , 46 lo spettro della scintilla; tutti gli altri sono spettri dell'arco cantante.

Ie costanti elettriche del circuito derivato sono segnate al margine (tav. II); e resta solo da avvertire che per i min. 26 e 54 l'intensità della corrente d'alimentazione fu 3 amp., mentre per tutti gli altri fu di 4 amp. circa.

Isa lunghezza dell' arco non fu, nei diversi casi, la stessa, sia perchè sarebbe stato difficile soddisfare a questa condizione, sia perchè, in corrispondenza alle diverse condizioni del circuito derivato, il resime oscillatorio si stabiliva in modo migliore, per lunghezze dell' arco diverse tra loro.

Le durate di posa, dipendentemente dalla diver'sa luminosità dell'arco e dalla sua stabilità, variavano fra 1 e 5 minuti; per gli spettri di scintilla occorsero invece delle pose molto piu lunghe. Nelle fotografie della prima serie, fu interposto sul caminino dei raggi un vetro debolmente colorato in giallo, per ottenere una maggiore uniformità $d$ impressione.

Nelia determinazione della lunghezza d'onda delle diverse righe (fatta, come si disse, per confronto con lo spettro solare), s'impiego la magnifica carta fotografica del Rowland ed una buona macchina a dividere Perreaux

Risultati. - Le fotografie riprodotte mostrano nettamente la trasformazione dello spettro d'arco in quello di scintilla, che avviene quando ai poli dell' arco elettrico si deriva un circuito dotato di grande capacità e d'induttanza trascurabile. Questa trasformazione avviene in modo graduale, quando, mantenendo costante e piccolissima l' induttanza del circuito derivato, si fa gradatamente crescere, a partire da uno o due microfarad, la capacità del condensatore.

Così nei num. 26 e 54 (capacitá due microf.), si vedono conservati tutti gli elementi caratteristici dello spettro d' arco, quali le bande 5632, 5165, 4737, 4382 del carbonio, e le bande del cianogeno 4604, 4216, quasi con la medesima intensità che 
hanno nell' arco; mentre d'altra parte cominciano a comparire alcune righe dello spettro di scintilla del carbonio, come le 513:3, 5145, 5151, 4267, fra le quali l’ultima già brillantissima, alcune righe dello spettro dell arią, fra le quali molto brillanti le 5676, 5006, 5011, 4242, 4237, 3995; e contemporaneamente si vedono rinforzate alcune righe delle impurezze metalliche, che nello spettro di scintilla sono più intense che in quelle dell' arco.

Nei num. 22, 53 (capacità 10 microf.), le bande del carbonio sono già molto indebolite, ed indebolite notevolmente sono anche le bande del cianogeno, specialmente il gruppo 46114 (la coda 3984 del gruppo 4216 è quasi completamente scomparsa) e predomina invece lo spettro di scintilla, con le righe del carbonio 5380 , 5648, 5662, oltre alle precedenti, divenute molto brillanti, con numerose righe dell' azoto, dell' ossigeno, con la 6563 dell idrogeno (ottenuta nello studio spettroscopico preliminare) e con alcune righe d'impurezze molto rinforzate, come la 5989 del sodio, che diventa molto slargata, e le 4227 e 3969 del calcio.

Con la capacità di 20 microf. (num. 24,52) si può dire, che lo spettro d arco si è interamente trasformato in quello di scintilla ${ }^{1}$ ): si conservano, infatti, debolissime traccie di alcune bande del carbonio (che, del resto, non sempre mancano completamente nello spettro di scintilla), e le bande del cianogeno, molto intebolite (che quasi sempre fanno parte dello spettro della scintilla).

Accrescendo da 20 a 30 microf. la capacità, lo spettro ottenuto rimane sostanzialmente lo stesso, salvo qualche leggera modificazione nell' intensità dei diversi elementi.

Se, in serie co! condensatore di grande capacità, si introduce un rocchetto dotato d'induttanza, lo spettro si modifica, e tenile a riprendere i caratteri dello spettro dell' arco continuo.

Cosi i num. 39, 55 (capacità 20 microf., induttanza 0,032 milliherny) mostrano notevolmente indebolite le righe del car-

1) Nelle fotografiie 43,46 non si possono osservare talune righe, specialmente le più snttuli del carbonio $\theta$ delle impurezze mutalliche: esse erauo debolissime a causa della piccolezza della scintilla. 
bonio, ancora più indebolite, ed in parte estinte, quelle dell'aria ; mentre sono tornate, o rinforzate, le bande dello spettro d'ar'co che erano scomparse, o indebolite. Nell' insieme, l' aspetto di questo spettro è molto simile a quello dei num. 26 e 54, che corrisponde ad una cápacità dieci volte più piccola.

Impiegando con la stessa capacità autoinduzioni ancora più grandi, lo spettro diventa quasi identico a quello dell' areo continuo, come mostrano le fotografie 36,56 e 37,57 , ottenute rispettivamente con le induttanze 0,693, 5,227 milliherny, nelle quali si vedono un po' più intense che nell' arco le bande del cianogeno, specialmente le 4216 , ed alcune righe d' impurezze, come le $3969,4227,4272$ del calcio.

Queste piccole differenze si osservavano nell' arco percorso da correnti t’onco-sinusoidali (cioè quando l'ampiezza delle oscillazioni era grande) e scomparivano del tutto quando, accorciando convenientemente l'arco si passara al regime sinusoidale puro ").

Studio elettrico. - Per poter passare alla discussione di questi risultati, è necessario dire poche parole sugli elementi elettrici principali del fenomeno dell' arco cantante, nelle condizioni in cui furono fatte le fotografie.

L' esame fu portato sulla forma, l' ampiezza ed il periodo delle correnti oscillanti nell'ar'co; e sui limiti di variazione della differenza di potenziale ai suoi poli.

Forma delle oscillazioni. - Fu determinata per mezzo di un tubo di Braun, eccitato da una macchina Toepler a 20 dischi.

La deviazione del fascio catodico era prodotta da un rocchettino attaccato in serie con l'ar'co. Questo rocchettino inevitabilmente alterava le condizioni del sistema elettrico, influenzando il fenomeno oscillatorio. Per ridurre al minimo questa perturbazione, si sacrifico, per quanto fu possibile, la

1) Sui diversi regimi delinaco cantante, cf. Corbino, Atti A. E. I., vol. VII, pagg. $597,1903$. 
sensibilità del metodo, impiegando un rocchetto di filo di rame di appena 2,9 microhenry d'induttanza ').

Gli spostamenti del cerchietto fluorescente erano osservati con uno spécchio girante.

La curva della corrente nell' arco, quando il circuito de. rivato non conteneva induttanza, era simile a quella della schema qui accennato: il tratto orizzontale corrispondeva alla posizione che aveva il cerchietto, quando il rocchettinc non era percorso da corrente, e quindi l'areo era spento; il tratto a forma di angolo acutissimo corrispondeva ad una cor. rente che attraversava l'arco nel senso stesso della corrente d'alimentazione.

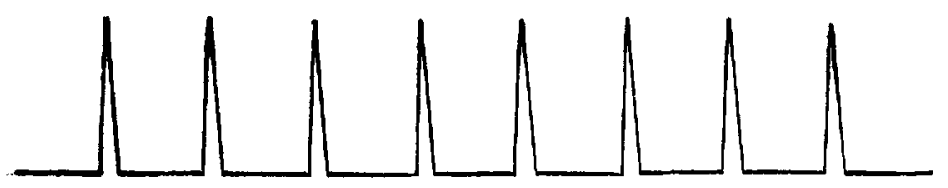

Fig. 2.

Da questo diagraınma risulta, che l' arco era acceso per un tempo brevissimo dalla corrente di scarica del condensatore, e si spegneva per un tempo notevolmente più lungo, durante il quale la corrente principale ricaricava il condensatore. Variando la capacita di questo, mutavano le durate di carica e di scarica e mutava anche il loro rapporto; e precisamente al crescere della capacitá, la prima cresceva più rapidamente della seconda.

Ampiezza delle oscillazioni. - Questo elemento, osservato anche col tubo di Braun, presentava delle variazioni repentine più o meno grandi, irregolari e senza cause apparenti, e delle variazioni regolari dipendenti, a parità delle altre condizioni, dalla lunghezza dell'arco; cosichè per ogni valore della capacità e della corrente d'alimentazione si aveva

1) Non pertanto ia presenza di questo rocehetto influenzava aneora il fenomeno oscillatorio come si desumeva dalla diminnzione, piccola nıa apprezzabile, dell'altezza del su'no. Con un amperometro termiro potei anche ricavare she $l^{\prime}$ intensità efficace nelJ'areo reniva diminuita di quasi un decimo $(0,10$ a 0,06$)$ per l'introdnzione del rocchettino. 
una lunghezza dell' arco optima, per la quale l' ampiezza era massima ${ }^{2}$ ).

Nel seguente specchietto sono riportate le ampiezze massime (A) in amp., relative ad una corrente d'alimentazione di 4 ampère '), alla capacità (C), all' induttanza dei soli attacchi ed alle lunghezze optimae (e); queste sono espresse in unità arbitrarie, e furono osservate, proiettando con una lente l' immagine dell' arco sopra una scala pellucida graduata.

I.

\begin{tabular}{c|c|c|c}
\hline $\mathrm{C}$ & $e$ & $\mathrm{~A}$ & $A / V \overline{\mathrm{C}}$ \\
\hline 30 & 7,5 & 172,5 & 31,5 \\
20 & 9 & 148,4 & 33,1 \\
10 & 10,5 & 110,4 & 34,5 \\
5 & 12,5 & 79,3 & 35,6 \\
2 & 9,5 & 48,3 & 34,3
\end{tabular}

I numeri dell' ultima colonna mostrano che le ampiezze massime, osservate per diffenti valori della capacitá, si mantengono, con buona approssimazione proporzionali alla radice quadrata della capacità. Le piccole oscillazioni del rapporto A $\boldsymbol{V}^{\prime}$ ' sono, probabilmente, imputabili ai mutamenti nei valori limiti della differenza di potenziale, dipendenti dai cambiamenti di lunghezza dell' arco; si rileva infatti un andamento quasi parallelo tra questo elemento e quel rapporto.

Differenza di potenziale ai poli dell' arco. Per queste determinazioni potè servire lo stesso tubo di Braun, perchè munito degli elettrodi piani per la deviazione elettrostatica. Le deviazioni accusate, anche per differenze di potenziali notevoli, erano piccole, e per poterle misurare fu necessario

1) Nel fare le fotografie deyli spettri, si cercava di mantenere sempre l'arco in queste condizionni di nassima anpiezza, e ciò si otteneva con l'apprezzamento dell'intensità del sunno.

2) Questa, per la capacità più piccola, era ugualo a 3 amp., come precedentemente fu anche arrertito. 
rendere stabile la posizione del cerchietto fluorescente, eccitando debolmente il tubo, e rjcorpere ad un cannocchiale munito di oculare micrometrico. Ad una differenza di potenziale di 150 volt, corrispondeva uno spostamento del cerchietto misurato da 10 giri e $\$ \frac{30}{100}$ del tamburo del micrometro.

Nel seguente specchietto sono riportati i limiti superiori delle differenze di potenziale ai poli dell' arco (potenziali di scarica) per le diverse capacità; $i$ limiti inferiori (potenziali residui) erano sempre di pochi volt, 10 all' incirca.

II.

\begin{tabular}{c|c}
\hline $\mathrm{C}$ & $\mathrm{v}$ \\
\hline 30 & 120 \\
20 & 125 \\
10 & 130 \\
5 & 110 \\
2 & 95
\end{tabular}

Introducendo in serie col condensatore di 20 microfarad il rocchettino d' induttanza 0,0029, o l'altro di 0,032 millihenry, i limiti della differenza di potenziale variavano pochissimo. Con l'induttanza 0,693 millihenry, il potenziale di scarica passava da 125 a 115 , e quello residuo da 10 a 15 circa. Con induttanze più grandi, detti limiti tornavano ad allontanarsi; cosi con 5,227 millihenry erano un poco più lontane che senza induttanza (regime tronco-sinusoidale esagerato).

Periodo. - Come precedentemente fu avvertito, questo elemento risultava di due parti distinte, di diversa grandezza e diversamente influenzate da un mutamento di capacità. Era molto difficile rilevare, dalle curve osservate nello specchio girante, la dipendenza esatta della durata di ciascuna parte dalla capacitá e dagli altri elementi elettrici ; principalmente a causa dell' incessante e irregolare variabilità del fenomeno oscillatorio. Peró si poteva nettamente apprezzare che in tutti i casi (con induttanza trascurabile) la durata della fase di 
scarica era minore della quarta parte della durata totale di un' oscillazione.

Dovetti perciò limitarmi ad una stima approssimativa della frequenza $n$, determinando con un sonometro l'altezza del suono che l'arco emetteva a preferenza. Ecco i numeri ottenuti variando la $\mathrm{C}$, e per $\mathrm{L}$ trascurabile.

III.

\begin{tabular}{c|c}
\hline $\mathrm{C}$ & $n$ \\
\hline 30 & 5100 \\
20 & 6200 \\
10 & 8000 \\
5 & 11000 \\
$\dot{\mathcal{Z}}$ & 20000
\end{tabular}

Determinai anche i numeri di oscillazioni che si avevano, introducendo in serie col condensatore delle induttanze. I seguenti sono quelli che si ebbero colla capacità 20 .

IV.

\begin{tabular}{c|c}
\hline I. & $n$ \\
\hline trascur. & 6200 \\
0,032 & 1800 \\
0,693 & 1000 \\
5,222 & 400
\end{tabular}

Per le considerazioni che in seguito dovremo fare, ci occorre conoscere un limite inferiore della potenza media spesa nell' arco durante ciascuna scarica del condensatore.

La potenza spesa nell' intero circuito è uguale a $\frac{\mathrm{C} v^{2}}{2 t}$, essendo $v$ la differenza tra il potenziale di scarica e quello 
residuo, e $t$ la durata della scarica. Le differenze di potenziale $v$, per quanto precedentemente fu osservato, possono ritener'si date in volt dai numeri riportati nella colonna $v$ del quadro seguente. Come durata della scarica assumeremo la quarta parte del periodo, cioè $\frac{1}{4 n}$, con ciò otterremo dei valori della potenza media più piccoli dei veri (e le differenze più notevoli corrisponderanno alle capacitá più grandi). D' altra parte la frazione dell' energia, dissipata nei brevi fili di congiunzione dell' arco con le armature del condensatore, era certamente molto più piccola della metà ${ }^{1}$ ).

Si può quindi affermare, che la potenza spesa nell' arco (per L trascurabile) doveva essere piu grande dei valori $\mathrm{P}_{m}=\mathrm{C} v^{\mathbf{2}} n$ scritto qui sotto, ed espressi in watt.

V.

\begin{tabular}{c|c|c}
\hline $\mathrm{C}$ & $v$ & $\mathrm{P}_{m}$ \\
\hline 30 & 110 & 1851 \\
20 & 115 & 1643 \\
10 & 120 & 1170 \\
5 & 100 & 550 \\
2 & 90 & 324
\end{tabular}

Come si rede, al crescere della capacita, la potenza media cresce sempre, però meno rapidamente di essa.

Furono anche calcolati dei limiti superiori delle potenze medie spese nell' arco quando il circuito derivato possedeva induttanza, per mezzo dell' energia del condensatore $(v$, ampiezza della differenza di potenziale sulle armature, fu misurata col metodo avanti letto) e del tempo di scarica ricavato dalla forma della curva d’intensità, osservata col tubo di

1) L'energia dissipata nel circuito non raggiunge la metà dell'energia complessira, anche nelle scariche oscillanti, quando la resistenza $\theta$ l' induttanza del circnito di scarica non sono grandi in paragone con la capacità. Cfr. Battelli e Magri, Nuoro Cimento, ser. 5a, vol. $3 \bullet$, pag. 262 . 
Braun, e dal valore della frequenza $n$. Nel quadro VI sono dati quelli relativi alle fotografie 39,$55 ; 36,56 ; 37,57$.

VI.

\begin{tabular}{c|c|c}
\hline $\mathrm{L}$ & $v$ & $\mathrm{P}_{n}$ \\
\hline 0,032 & 115 & 620 \\
0,613 & 105 & 300 \\
5,222 & 100 & $\left.110^{\prime}\right)$
\end{tabular}

La comparsa di alcune righe dello spettro di scintilla in quello dell' arco, sotto determinate condizioni, è stata già varie volte osserva:a.

I sigg. Hartmann ed Eberhard ${ }^{3}$ ) notarono questo fenomeno negli spettri dell' arco f'x elettrodi di silicio, magnesio, zinco, cadmio, alluminio, quando esso veniva prodotto sott' acqua, o in una co'rente d'idrogeno ; ed attribuirono tale modificazione spottrale alla presenza di questo gas nel cammino attraversato dalla corrente.

Lo stesso Hartmann, in uu lavoro successivo ${ }^{3}$ ), dimostrò la presenza di cerie righe di scintilla, nello spettro di un arco alimentato con deboli correnti, e prodotto fra elettrodi di ma. gnesio, di zinco, bismuto e piombo nell' aria ordinaria. Esse si rinforyavano nutevolinente, quando si ficera diminuire l'intensitá della corrente di alimentazione; così p. es. l'intensità della riga 4481 del milgnesio (speitro di scintilla) crebbe da 0,03 a 10, quando l'intensilà della corrente nell' arco decrebbe da 8 a 0,4 ampere. $L^{\prime} A$. vide in questi fatti una conferma della sua opinione che, causa della modificazione fosse

1) Queston vulore è più pirenlo dalla pntenza fata dal circuito di alimentazione, perchè laren era periorso da roriente anche per buona parte del tempo di carica.

9) Hartmann ed Eburhard, Beri. Ber., piag. 40, 1903.

3) Hartmaun, Ber\}. Ber., pag. 234, 1903. 
un' azione dell' ambiente (in questo caso molto povero di vapore) sulle particelle emittenti (ioni), e che la temperatura dell' arco non dovesse influire sulla natura dello spettro emesso; concludendone che l'emissione dell' arco dovesse consistere in un fenomeno di elettroluminescenza.

Poco tempo dopo, il sig. Crew ${ }^{1}$ ) osservò la comparsa di alcune righe di scintilla nello spettro dell' arco intermittente proloito fra elettrodi di magnesio, nell' aria ambiente ed in altre atmosfere gassose, tutte le volte (e solo allor'd) che un' elevata e rapidanente variabile f. e. $\mathrm{m}$. di autoinduzione veniva destata, o soffiando l'ar'co, o modificando l'induttanza del circuilo d'alimentazione. E ritenne, che la comparsa di righe di scintilla, nello spettro dell' arco prodotto sott' acqua o in una corrente d'idrogeno fosse da attribuire al fatto, che in queste atmosfere si producono facilmente variazioni molto rapide della tensione ai poli dell' arco e della corrente che lo attraversa.

Recentemente i sigg. Fabry e Buisson ${ }^{2}$ ) hanno notato la presenza di righe di scintilla nello spettro d' arco del ferro e di qualche altro metallo (nikel, rane), nelle immediate vicinanze dei crateri. Fssi ritengono che la condizione di emissirne di queste righe sia l'esistenza di una velocità grandissima delle particelle emittenti, dovuta alla presenza di un campo elettrico intenso, o forse al movimento di agitazione termica.

Riassumendo: si puó ritenere, che tracce delle righe di scintilla si trovano presenti nelle regioni dell' arco immediatamente vicine ai due crateri; tali righe, peró, si notano in tutto l'arco, sia quando è alimentato da una corrente debole (e sono tanto più intense quanto più debole è questa); sia quan to è soggetto a brusche ed intense variazioni di f. e. m; sia quando è prodotto in particolari atmosfere. Le cause che producono queste modificazioni (o forse la causa unica) sono mal definite dai diversi autori, e si possono riguardare come sconosciute

1) Crew, Astroph. Journ., 20, pag. 274, 1904. (Visto in sunto in mancanza dell'origunale.

2) Fabry \& Buisson, C. R., t. CXLVI, pag. 751, 1908. 
Meglio studiata è la trasformazione inversa e graduale dello spettro di scintilla in quello d'arco.

I primi lavori su questo importante argomento sono dovuti ai sigg. Schuster ed Hemsalech, e specialmente a quest' ultimo. In essi sono minutamente esaminate le modificazioni successive, che subisce lo spettro della scintilla (ordinaria) prorlotta in un circuito con capacita notevole e piccolissima induttanza, quando quest' ultimo elemento si fa crescere gradatamente. I risultati di questi studi, rimasti classici, sono a tutti noti ed a me basta ricordare, che in complesso la modificazione prodotta dall' aumento dell' induttanza, tende ad avvicinare sempre più lo spettro della scintilla a quello dell'arco, prodotto fra gli stessi metalli.

L'Hemsalech ${ }^{1}$ ) spiegò la trasformazione spettrale, come un effetto combinato della diminuzione dell' energia spesa nella scintilla, e dall' aumento della durata delle oscillazioni; in conformità del concetto che si era formato del fenomeno della scarica. Secondo questo concetto, la prima oscillazione passerebbe attraverso all' aria interposta fra gli elettrodi, rendendola incandescente; le oscillazioni successive attraversereb. bero il vapore che si svolge (allo stato incandescente) dagli elettrorli, diffondendosi nello spazio fra essi compreso; questa porzione di vapore più riscaldata dalle oscillazioni emetterebbe le righe corte, caratteristiche dello spettro di scintilla (ritenute quindi di più alta temperatura); la porzione rimanente, che costituisce l'aureola, emetterebbe le righe lunghe, appartenenti allo spettro di arco.

In armonia con ciò egli ritenne:

che la scomparsa delle righe dell' aria, prodotta dall' accrescimento dell' induttanza, fosse dovuta alla diffusione del vapore, che si svolge più copioso dagli elettrori, nel cammino attraversato dalla scarica, per effetto della più lunga durata di ciascuna oscillazione;

che la scomparsa delle righe corte del vapore dipen. desse dall' abbassamento di temperatura, che necessariamente

1) Hemsaioch, Rech. exp. sur le spectre átine. Hermnon, Paris, 1901. 
devono trarsi dietro l' allungamento del periodo, e la diminuzione dell' energia spesa nella scintilla;

e che il rinforzarsi delle righe d'arco fosse doruto ad uno accrescimento della temperatura e della densità dell' aureola; sempre in grazia della maggior durata di tutto il fenomeno.

Il Prof. Puccianti ${ }^{1}$ ), in una sua pregevole Memoria, modifica queste conclusioni, fondandosi sopra una diversa interpretazione del fenomeno della scarica oscillante, ricavata dall' esame delle belle fotografie della scintilla, ottenute dai professori Battelli e Magri ${ }^{2}$ ).

Egli riguarda i fenomeni luminosi, che hanno luogo in seno al gas ed al vapore, come profondamente diversi. Considera l'emissione del gas come un fenomeno di Juminescenza elettrica, che segue fedelmente l'andamento della scarica nella prima o nelle prime oscillazioni, e che cessa nelle successive, a causa forse del progressivo aumento di temperatura della massa emittente. Attribuisce invece ald un effetto di elerata temperatura l'emissione del vapore, perchè trova che essa non segue lantamento della scarica, non viene ravivata dalle scariche parziali successive e si spegne lentamente, potendo ancora durare alcuni milionesimi di secondo, dopo che la scarica è cessata.

Egli spiega la modificazione prodotta da un aumento dell' induttanza nello spettro del vapore, come il risultato di due effetti antagonisti : la diminuzione dell' energia dissijata nella scalica, che tende ad indebolipe tutte le righe emesse; lo sviluppo più copioso di vapore, che ne accresce la densità, e quindi l'intensità della luce emessa. Sulle righe corte, che anche per questo A. sono di più elevata temperatura, dovrebbe prevalere il primo effetto, sulle lunghe il secondo.

Altri lavori sugli spettri delle scariche oscillanti sono dovuti ai sigg. Berndt, Schenk, Neculcea, King ${ }^{3}$ ).

1) Puccianti, N. Cim, ser. 5a, vol 9, 1905 .

2) Battelli e Magri, 1, c., oppure Ace. Seienze Torino: Mem, 2, 51 1905.

3) Berndt, Diss. Halle, 19ı1; Sehenk, J. Hopk. Un Cire, 152. pag. 79, 1901 ; Nèculcèa, C. R., 134. pag. 1494, 1902 ; id., Bull. Bukarest, par. 381, 1902 ; id., C. R., 135, pag. 25 1903: King, Astroph. Journ., 39, pag. 225, 1904. 
Berndt ricercò se l'aumento di resistenza produceva modificazioni spettrali analoghe a quelle osservate con l'atumento d'indultanza, conlormemente all'opinione già espressa du Hasselberg; e trovò che gli spettri ottenuti con una grande induttanza e con la resistenza equivalente (quella per la quale la scarica unilaterale presentara lo stesso massimo di corrente della scarica oscillante) erano presso che simili. Concluse ammettendo che la causa della modificazione risiedeva, in entrambi i casi, nell' abbassamento di temperatura, dipendente dalla diminuzione dell' intensitd massima e dall' aumento della durata della scarica.

Fra i lavori rimanenti, il più notevole è quello di Schenk ${ }^{1}$ ), sia per i mezzi impiegati nello studio spettrale, sia, e più ancora, per le conclusioni tratte, che sono in completo contrasto con quelle di Hemsalech ed altri.

Egli ammise che il rapore metallico possa raggiungere facilmente una temperatura più elevata, quando le oscillazioni vengono rallentate per mezzo di una più grande induttanza, perchè allora ha più tempo per riscaldarsi; e che per conseguenza lo spettro dell' arco debba corrisponitere ad una temperatura più elevata dello spettro di scintilla.

Riportiamo, infine, l'opinione manifestata-dal prof. Kayser ${ }^{2}$ ) su questa complessa quistione. Egli pensa, conformemente all'idea primitiva dell' Yeinsalech, ed a quella fortemente sostenuta, per il caso dei gas, dai sigg. Richard e Trowbridge, che il mutamento dell' induttanza non interviene nella modificazione spettrale, principalmente per mezo di un mutamento di temperatura, ma per un'azione diretta della scarica sul gas, o vapore, che essa attraversa; azione che dipenderebbe intimamente dalle circostanze elettriche della scarica, e quindi dalle costanti del circuito in cui avviene.

In altri termini, seconilo il concetto del Kayser, il mutamento di temperatura può magari essere una causa concomitante. che agevoli la trisformazione spettrale; ma sopra tutto il cambiamento d'induttanza leve influire in modo diretto, alterando l' andaimento della scarica.

1) Cfr. Kuyser, Handt. der Spectr., II, pp. 172-173.

2) Kayser, l. c. 
Riassumendo: secondo l'opinione prevalente, la causa principale della modificazione spettrale, prodotta da un aumento d'indutanza, risladerebbe in un mutamento della temperatura del corpo emittente, perchè l' emissione nella scintilla e nell'arco arrebbe or'igine termica; ma la tenıeratura della scintilla sarebbe maggiore - secondo alcuni - minore - secondo altri - di quella dell' arco.

Secondo un' altı a opinione (Kayser), l'emissione avrebbe origine diversa nella scintilla e nell' arco; in quella si ridurrebbe ad un fenomeno di elettroluminescenza, in questo ad un fenomeno termico. La causa principale delle morfificazioni, prodotte dall induttanza, dor rebbe risiedere nel cambiamento delle circostanze elettriche della scarica.

Secondo un' ultima opinione (Puccianti), sarehbero distinte non solo le origini dell' emissione nei due fenomeni scintilla, arco; ma anche quelle dell' emissione del gas e del vapole nella medesima scintilla; il primo emetterebbe per elettroluminescenza, il secondo per temperatura. La causa delle modificazioni dipenderebbe da questo elemento.

Le nostre ricerche credo possano servire a rischiarare in qualche punto questa complessa quistione dell' emissione nella scintilla elettrica.

Il risultato più immediato di esse, ci induce a limitare I' importanza attribuita, alle circostanze puramente eletriche (e particolarmente alle oscillazioni) che intervengono nell' emissione.

Noi abbiamo ottenuto, infatti, uno spettro coincidente con quello della scintilla ordinaria, benchè il nostro fenomeno fosse da questa essenzialmente diverso. La scarica, nel caso nostro, s' iniziava con unir differenza di potenziale, anzi, più esattamente, cou un campo elettrico assi più debole di quello occorpente per la scintilla; era sempre unilaterale, ed aveva una durata molto maggiore del periodo d'oscillazione delle comuni scintille. (A questa stessa conclusione portano l' esperienze di Berndt precedentemente citate). 
Le nostre ricerche contraddicono, poi, in modo evidente le opinioni del sigr. Schenk. In primo luogo, se veramente lo spettro di scintilla corrispondesse ad una temperatura minore di quella dell' arco, in modo che la modificazione spettrale, prodotta da un accrescimento d'induttanza, dipendesse dal maggior tempo che al vapore è concesso per raggiungere un' elevata temperatura, in grazia dell' accresciuta durata delle oscillazioni, non avremmo dovuto ottenere lo spettro di scintilla, nelle condizioni delle nostre esperienze.

Infatti, essendo stata in questo caso la durata della scarica sempre maggiore del periodo d'oscillazione e della durata complessiva di una comune seintilla, il vapore avrebbe potuto sempre raggiungere quella temperatura elevata, a cui corrisponderebbe, secondo lo Schenk, lo spettro d'arco.

Ma volendo anche ammettere che, per la discontinuità dell' accensione, l'arco rimanesse, nelle nostre esperienze, ad una temperatura inferiore a quella dell' arco contınuo, avremmo dovuto ottenere lo spettro di scintilla, di preferenza nel caso di scariche più brevi, cioè con le capacità più piccole.

E vi ha di più. Lo spettro di scintilla subiva modificazioni identiche, che lo avvicinavano allo spettro d'arco, tanto in. troducendo in serié col condensatore di grande capacità una induttanza, quanto diminuendo convenientemente la capacitå di questo; vale a dire, tanto accrescendo quanto diminuendo la durata della scarica.

Nè si puó ammettere che, nel caso in cui la capacità veniva diminuita, il vapore o gas raggiungesse una più elevata temperatura, perchè l'energia disponibile era spesa in un tempo più piccolo. I numeri riportati nel quadro $\mathrm{V}$ ci dicono chiaramente che, diminuendo la capacitá, diminuiva la potenza media spesa nel cammino della scarica.

Questo rilievo ci porta ritenere, che la causa principale della modificazione spettrale, prodotta da un aumento dell’induttanza, o da una diminuzione della capacita, risieda appunto nella diminuzione, che avveniva in entrambi i casi, della potenza media spesa nel cammino della scarica.

In questa opinione, siamo fortemente confermati dalla seguente osservazione: Iat potenza media spesa nell' arco delle 
nostre esperienze (per le condizioni in cui si osservava lo spettro di scintilla) era, verosimilmente, dello stesso ordine di grandezza di quella spesa in una ordinaria scintilla oscillante. Per questo raffronto ci possiamo servire in primo luogo della bella Memoria dei proff. Battelli e Magri, precedentemente citata, dalla quale abbiamo tolto i numeri riuniti nel segnente quadro, che si riferiscono a scintille della lunghezza di 1,5 mm., cioè all'incirca della stessa lunghezza dell' arco elettrico da noi studiato. Nella prima e nella seconda colonna, sono riportati i numeri che danno le induttanze e le capacità in cm.; nella terza, i periodi in secondi; nella quarta il numero totale di oscillazioni $K$ per ciascuna scarica apprezzabili nelle rispettive fotografie; nella quinta le quantitá di calore spese nella scintilla, in piccole calorie; e nella sesta, infine, abbiamo scritto i valori della quantità :

$$
\mathrm{P}_{m}=\frac{4,18 \mathrm{Q}}{\mathrm{K} \mathrm{T}}
$$

che, nell' ipotesi in cui l'energia svolta durante le K oscillazioni visibili differisca poco dalla totale, rappresenta la potenza media consumata, espressa in Watt.

\begin{tabular}{|c|c|c|c|c|c|}
\hline $\mathbf{L}$ & $c$ & $\mathrm{~T}$ & $\mathbf{K}$ & $Q$ & $P_{m}$ \\
\hline 74140 & 14175 & $6,79 \cdot 10^{-6}$ & 30 & 0,016 & 325 \\
\hline 29470 & 14175 & $4,30 \gg$ & 16 & 0,020 & 1200 \\
\hline 29470 & 7168 & $3,04 \gg$ & 12 & 0,012 & 1400 \\
\hline 29470 & 3568 & $2,15 \gg$ & 12 & 0,006 & 970 \\
\hline 17460 & 3568 & $1,70 \gg$ & 8 & 0,008 & 2420 \\
\hline 3669 & 3568 & $0,80 \gg$ & $8 ?$ & 0,012 & $7500 ?$ \\
\hline
\end{tabular}

Se facciamo il calcolo per una scintilla sempre della lunghezza 1,5 mm., prodotta nelle condizioni sperimentali delle fotografie $43,46(\mathrm{C}=1000 \mathrm{~cm}$. $\mathrm{L}$. $=3000 \mathrm{~cm}$. circa, $\mathrm{V}=6000$ volt), deducendo per confronto con i risultati della medesima Memoria dei sigg. Battelli e Magri un limite minimo di $\mathrm{K}$ e del- 
l'energia dissipata fuori della scintilla, otteniamo come limite superiore di $\mathrm{P}_{m}$ il valore 2300 watt. Un calcolo analogo applicato alle condizioni sperimentali di Hemsalech, for nisce valori di $P_{m}$ compresi fla 3000 e 100 watt: il più grande di essi si riferisce alla più piccola induttanza impiegata (primo strato del rocchetto A) "), nel qual caso lo spettro era identico, o quasi, a quello che si aveva con l'induttanza dei soli attacchi (sconosciuta); l'ultimo si riferisce all induttanza massima adoperata (molto grande, 0,07 henry circa!), nel qual caso lo spettro presentava solo righe darco o di fiamma.

Questa coincidenza, rimarchevole se la lunghezza della scintilla è la stessa di quella dell'árco da noi studiato, più non sussiste per lunghezze diverse della scintilla. Al variare della lunghezza varia la massa gassosa eccitata dalla scarica, ed è naturale che varî pure la potenza media necessaria per quel grado di eccitazione.

Siamo per tal via indotti a pensare che la natura dello spettro emesso da un vapore o gas attraversato dalla scarica (e forse più generalmente eccitato in un modo qualsiasi) debba dipendere essenzialmente dalla potenza media spesa nella unità di massa, e che limitata e secondaria debba essere la influenza del modo con cui tale potenza viene introdotta.

Per rafforzare questo concetto sarebbe stato necessario un confronto fra le masse gassose eccitate nell'arco delle nostre esperienze e nelle scintille scelte a confronto. Purtroppo non possediamo, in riguardo, alcun dato sicuro su cui potere appoggiare le nostre considerazioni; ma possiamo bene affermare che l' arco, nelle nostre esperienze, doveva avere una sezione notevolmente più piccola dell'arco continuo, per la discontinuita dell'accensione, e la brevità di ciascuna scarica. La potenza disponibile veniva, quindi, consumata in una pic-

1) Cfr. Hemsalech Men. c., pag. 28, 47 e seg. - E necessario avvertire che i valori numerici delle iniluttanze dati a pag. 98 non soun esutti. Il calcolo dallo pntenze molio farto enn questi datj mi aveva fornito numeri di un ordine di grandezza assai maggiore di qutello aspettato (limite superiore 60,010). Rifarendo il calcolo delie induttanze con la formoia $L=\frac{4 x^{2} w^{2}, 3}{l}$, usata dall'A. e con i dati orcorrenti che si trovano nella Memoria stessa ho potuto rilevare l'errore materiale dell'Hemsalech (per $n$ ora stato posto il uumero di spire per em. invece del numero totale). 
cola massa di gas, che poteva raggiungere un'eccitazione elevata.

Una conferma dello stesso concetto risulta dal completo parallelismo, che nelle nostre esperienze si puó osservare, tra la potenza media impiegata e la struttura dello spettro; e dalla indipendenza rimarchevole di questa dai particolari valori delle costanti elettriche del circuito derivato. Così le fotografie riprodotte, che sono ordinate col criterio della maggiore affinitá di struttura, risultano anche ordinate secondo la potenza impiegata; e si puó notare, per es., che i due spettri 39,55 e 26,54, assai simili tra loro, corrispondono a condizioni del circuito elettrico molto diverse; tanto che i periodi stavano come 1 a 11, mentre le potenze impiegate nei due casi erano 620 e 324 , cioè molto vicine. ( $E$ si badi che il primo si accosta più del secondo allo syettro di scintilla).

E poichè in queste esperienze si può ammettere (trattandosi sempre del medesimo fenomeno) che la massa gassosa eccitata non variava notevolmente da un caso all altro, e che quindi la potenza specifica cresceva insieme con la protenza complessiva, risulterebbe da esse che un legame intimo esiste veramente, tra la potenza media specifica impiegata nella scarica e la struttura dello spettro emesso.

Un tale modo di vedere, può spiegare anche il comportamento offerto dai gas. Lo spettro a righe di questi, corrisponderebbe ad un grado molto elevato di eccitazione (cinè avrebbe bisogno dell'impiego di una grande potenza) uguale, e for'se più elevato di quello necessarjo per le righe corte dei vapori. Per questa ragione le righe del gas si lasciano osservare solo con scariche (condensate) di breve perjodo, e nelle sole prime oscillazioni; mentre con scariche più lente, o con oscillazioni poco ampie, il gas emetterebbe lo spettro di bande, che sarebbe perciò di più bassal eccitazione.

Lo stesso modo di vedere si adatta bene anche ai fenomeni osservati nell' arco:

Se si vuole infrodurre in un arco a corrente continua una grande potenzà, è necessario far crescere l' intensità della corrente; con che crescono, quasi proporzionalmente, l'area dei crateri e la sezione trasversale della colonna di vapore; 
e quindi la massa dell' aeriforme eccitato $\left.{ }^{1}\right)$. E poichè al crescere dell'intensità della corrente, la differenza di potenziale ai poli dell'arco (e agli estremi della sola colonna positiva), va decrescendo - molto rapidamente per le intensità piccole, poco per le grandi - vale a dire, poichè la potenza spesa nell' ar'co cresce meno rapidamente dell' intensitá della corrente (specialmente per le intensitá piccole), se ne deve concludere che la potenza specifica, spesa in un arco a corrente continua, diminuisce, al crescere dell' intensità ; e la diminuzione dev' essere particolarmente apprezzabile per le intensità piccole, poco o punto per le grandi.

E da quanto precedentemente si è esposto si dedurrebbe che al crescere dell' intensità della corrente d'alimentazione dell'arco, a partire dai valori più piccoli possibili, lo spettro deve andare modificandosi, denotando gradi di eccitazione via via meno elevata; e ció in modo particolarmente, o solamente, apprezzabile per le piccole intensità.

Questa deduzione è manifestamente conforme alle esperienze del sig. Hartmann, precedentemente riferite, nelle quali furono osservate righe di scintilla in un arco alimentato con deboli correnti, nonchè a quelle del sig. Huff ${ }^{2}$ ), nelle quali non si potè riscontrare alcun mutamento di struttura nello spettro di un arco, fra carboni omogenei o animati, facendo crescere la corrente da 2 a 250 ampère.

Anche i fatti osservati da Crew, da Hartmann ed Eberhard e da Fabry e Buisson trovano facilmente una spiegazione col nostro modo di vedere.

Per i primi, s'intende bene come un'intensa f. e. m. di autoinduzione possa produrre quell'aumento istantaneo di potenza specifica, che sarebbe la causa della temporanea comparsa di righe di scintilla.

Per $i$ secondi, basta ricordare che un arco a corrente continua, in un ambiente termicamente più conduttore (come l'acqua e l'idrogeno), ha bisogno di una maggiore densità di corrente e di una più grande differenza di potenziale che

1) Com'è noto la densità della corrente varia pocbissimo al crescere dell'intensità.

Cfi. J. Stark. Drude Ann., vol. 12, pay. 690. 1903.

2) Huff, Astroph. Journ. 16, pag. 27, 1902. 
l'arco nell'aria; di modo che, nel primo la potenza specifica è maggiore che nel secondo, e perciò negli strati più interni del primo possono comparire righe di eccitazione più elevata, che non si osservano nel secondo.

E finalmente per gli ultimi possiamo osservare, che in vicinanza degli elettrodi, esiste sempre una brusca caduta di potenziale, a cui deve necessariamente corrispondere una potenza specifica sviluppata più grande che in tutto il resto dell'arco, e quindi un grado di eccitazione più elevato.

Riassumendo, possiamo concludere che:

lo spettro di scintilla si può ritenere corrispondente ad un grado di eccitazione più elevato di quello a cui corrisponde lo spettro d'arco;

la trasformazione graduale del primo spettro nel secondo, e riceversa, dipende essenzialmente dalle variazioni della potenza media specifica, spesa nel cammino percorso dalla corrente;

il valore di questa potenza può, per questa trasformazione (e forse in generale), considerarsi come il parametro unico, che determina ciascuna struttura spettrale.

Queste conclusioni non pregiudicano la questione, tanto dibattuta, dell'origine dell' emissione; nulla infatti esse ci dicono che permetta di decidere, se la potenza necessaria per produrre una data struttura spettrale, e fornita sotto forma elettrica, si trasformi direttamente in energia luminosa, ovvero se assuma un' altra forma, per es. la termica, prima di dare origine all'emissione stessa.

Se non che, per mezzo di apposite esperienze, delle quali ho già annunziato i risultati sui " Comptes rendus de l'Acad. des Sciences ${ }^{1}$ ), ho mostrato che nell' arco musicale, come nella scintilla, si possono ottenere effetti termici di grado più elevato che nell' arco continuo. Difatti sono riuscito con l'arco musicale a fondere insieme delle particelle di carbonio; mentre coll' arco ordinario non ho potuto constatare traccia di

1) Fasc. $8^{\circ}$, pag. 475 ; fasc. $10^{\circ}$, pag. $616,1909$. 
tale fatto, come non ne potè constatare Moissan, nella lunga serie delle sue ricerche sul forno elettrico.

Si può dunque ritenere, non solo che l'emissione della scintilla e dell' arco musicale si ottiene sotto l'impiego di una potenza media specifica più grande di quella impiegata per l'arco ordinario, ma anche che tale emissione è accompagnata da una temperatura più elevata di quella dell' arco.

Noi veniamo così a rafforzare il concetto dell'esistenza di un legame intimo fra temperatura ed emissione luminosa, $\theta$ con ciò l'ipotesi che su esso si appoggia, dell'origine termica dell' emissione.

Ma del resto, queste distinzioni hanno perduto oggidì gran parte dell'importanza che fu loro attribuita '); dopo che nei lavori di Smithells e di Stark, si è cercato di dare un significato preciso alle incerte espressioni di luminescenza elettrica e chimica.

Con l'introduzione dei concetti di temperatura elettrica e parziale, la causa dell' emissione viene, in fondo, ad essere ricondotta alla forza viva che possiede la particella emittente (träger) nell' istante in cui il fenomeno luminoso si produce: cicè, alla medesima causa che produrrebbe l'emissione di natura termica. In tal modo è stato esteso, solamente, il concetto di temperatura ai träger, avvertendo che per essi si deve tenere in computo la velocità complessiva da cui sono animati abbia essa, o no, una direzione preferita.

In altri termini, l'emissione, in tutti e tre gli aspetti nei quali è stata finora distinta, avrebbe un origine unica: cinetica; lo spettro emesso dovrebbe, quindi, essere un indice diretto dalla forza viva posseduta da quei ioni che danno luogo al fenomeno luminoso.

Questi concetti armonizzano perfettamente con i risultati e le vedute da noi sopra esposti.

Al chiarissimo prof. Macaluso vada l' espressione della mia gratitudine, per l'opera benevola che ha speso a mio vantaggio.

1) Cfr. Puccianti. Degli spettri di righe. N. Cim., ser. 5.a, rol. XV, p. 95, 1908. 